\title{
STABILITY AND BIFURCATION ANALYSIS FOR A TWO-COMPETITOR/ONE-PREY SYSTEM WITH TWO DELAYS
}

\author{
GuO-Hu Cui and Xiang-Ping Yan ${ }^{\dagger}$
}

\begin{abstract}
The present paper is concerned with a two-competitor/oneprey population system with Holling type-II functional response and two discrete delays. By linearizing the system at the positive equilibrium and analyzing the associated characteristic equation, the asymptotic stability of the positive equilibrium and existence of local Hopf bifurcations are investigated. Particularly, by applying the normal form theory and the center manifold reduction for functional differential equations (FDEs), explicit formulae determining the direction of bifurcations and the stability of bifurcating periodic solutions are derived. Finally, to verify our theoretical predictions, some numerical simulations are also included at the end of this paper.
\end{abstract}

\section{Introduction}

During the last decades, two-species prey-predator systems described by ordinary differential equations (ODEs) have been proposed and studied extensively by many authors (e.g., $[4,5,14,19])$ since the pioneering theoretical works by Lotka [10] and Volterra [20]. However, there are maybe two species in some habitat and they coexist upon a single prey. Then in this case the growth dynamics about these species should be described by a three-species system. Hsu, Hubbell and Waltman [8] proposed the following two-competitor/one-prey model with a Holling type-II functional response

$$
\left\{\begin{array}{l}
u^{\prime}(t)=r u(t)\left(1-\frac{u(t)}{K}\right)-\frac{a u(t) v(t)}{1+b u(t)}-\frac{A u(t) w(t)}{1+B u(t)} \\
v^{\prime}(t)=v(t)\left(-d+\frac{e u(t)}{1+b u(t)}\right) \\
w^{\prime}(t)=w(t)\left(-D-G w(t)+\frac{E u(t)}{1+B u(t)}\right)
\end{array}\right.
$$

Received July 10, 2010; Revised March 10, 2011.

2010 Mathematics Subject Classification. 34K13, 37G05, 37G15, 92D25.

Key words and phrases. three-species system, time delays, bifurcation, stability, periodic solution.

†Supported by the National Natural Science Foundation of China (10961017) and "QingLan" Talent Engineering of Lanzhou Jiaotong University (QL-05-20A). 
where the variable $u(t)$ is the population density of prey species, and $v(t)$ and $w(t)$ are the population densities of two competitors; $r, K, a, b, A, B, d, e, D, G$ and $E$ are all positive constants. By constructing a suitable Lyapunov function, Ruan et al. [16] obtained the sufficient conditions for the global stability of the positive equilibrium of the system (1.1). For the wide studies of the system (1.1), the reader can refer the references $[2,3,13,17]$.

In proposing the model (1.1), however, the maturation time and the intrinsic growth time of each species are ignored. In fact, many species need to take certain time to become reproductive and mature. Therefore, it is often reasonable to incorporate different time delays into ODE systems to consider the maturation time and the intrinsic growth time of each species. In recent years, the effects of time delay due to maturation of the predator on the dynamics of predator-prey models have been studied by a number of authors (see, for example, [11, 18, 21, 12, 25] and references cited therein). But most of them investigated only the cases when the considered models have a single delay. From the viewpoint of biology and bifurcation, it is often necessary to consider different delays and multiple species. Based on these facts, a more reasonable model corresponding to the model (1.1) should be described by the following delayed differential system

$$
\left\{\begin{array}{l}
u^{\prime}(t)=r u(t)\left(1-\frac{u(t)}{K}\right)-\frac{a u(t) v\left(t-\tau_{1}\right)}{1+b u(t)}-\frac{A u(t) w\left(t-\tau_{1}\right)}{1+B u(t)} \\
v^{\prime}(t)=v(t)\left(-d+\frac{e u\left(t-\tau_{2}\right)}{1+b u\left(t-\tau_{2}\right)}\right) \\
w^{\prime}(t)=w(t)\left(-D-G w(t)+\frac{E u\left(t-\tau_{2}\right)}{1+B u\left(t-\tau_{2}\right)}\right)
\end{array}\right.
$$

where $\tau_{1} \geqslant 0$ denotes the time from birth to predation for two competitors and $\tau_{2} \geqslant 0$ represents the intrinsic growth time of prey species from birth to being a predated object.

Delayed models similar to (1.2) have been studied widely by many authors and some interesting results have also been obtained (see $[1,15,9]$ ). For example, Boudjellaba and Sari [1] investigated the loss of stability by the increase of delay in a three-dimensional competition model. Patra, Maiti and Samanta [15] considered the boundedness and stability in a delayed food chain model with Michaelis-Menten type ratio-dependent functional responses. Kar and Batabyalb [9] obtained the persistence and stability of a two-prey-one-predator system in the presence of a time delay due to gestation. It is well known that studies on dynamical systems not only involve a discussion of boundedness, stability and persistence, but also involve many dynamical behaviors such as periodic phenomenon, bifurcation and chaos. In particular, the properties of periodic solutions are of great interest arising through Hopf bifurcations in delayed systems, see Liu and Yuan [11], Wei and Ruan [21], Wei and Li [22] and Xiao and $\mathrm{Li}[23]$.

In this paper, we devote our attention to the effects of delays on the dynamical behavior of the system (1.2). That is to say, we shall choose the sum $\tau$ of 
the two delays $\tau_{1}$ and $\tau_{2}$ as the main parameter and show that when $\tau$ passes through a certain critical value, the positive equilibrium loses its stability and a Hopf bifurcation will take place. In particular, by using the normal form theory and the center manifold reduction for FDEs, the formulae determining the direction of Hopf bifurcations and the stability of bifurcating periodic solutions are also obtained.

The organization of this paper is as follows. In Section 2, by analyzing the characteristic equation of the linearized system of the system (1.2) at the positive equilibrium, the local stability of the positive equilibrium and existence of local Hopf bifurcations are addressed. In Section 3, by using the normal form theory of retarded functional differential equations developed by Hassard, Kazarinoff and Wan [7], the direction of the Hopf bifurcation and the stability of bifurcating periodic solutions are determined. Moreover, in order to verify our theoretical results, some numerical simulations are also included in Section 4 .

\section{Stability of positive equilibrium and existence of local Hopf bifurcations}

In this section, by analyzing the characteristic equation of the linearized system of the system (1.2) at the positive equilibrium, we investigate the stability of the positive equilibrium and the existence of the local Hopf bifurcations occurring at the positive equilibrium.

Respectively, define $u^{*}, v^{*}$ and $w^{*}$ by

$$
\begin{aligned}
u^{*} & =\frac{d}{e-b d} \\
v^{*} & =\frac{1+b u^{*}}{a}\left[r\left(1-\frac{u^{*}}{K}\right)-\frac{A w^{*}}{1+B u^{*}}\right], \\
w^{*} & =\frac{1}{G}\left(-D+\frac{E d}{e+B d-b d}\right)
\end{aligned}
$$

It is easy to see that if the condition

$$
\frac{e D}{E-B D+b D}<d<\frac{e}{b} \text { and } r\left(1-\frac{u^{*}}{K}\right)-\frac{A w^{*}}{1+B u^{*}}>0
$$

holds, then $u^{*}, v^{*}$ and $w^{*}$ given by the above expressions are all positive and thus the system (1.2) has a unique positive solution $E^{*}\left(u^{*}, v^{*}, w^{*}\right)$.

We are only interested in the positive equilibrium $E^{*}\left(u^{*}, v^{*}, w^{*}\right)$ since the model (1.2) is a mathematical biology model. Making a change of variables $u_{1}(t)=u(t)-u^{*}, u_{2}(t)=v(t)-v^{*}, u_{3}(t)=w(t)-w^{*}$, the system (1.2) can 
be transformed into the following equivalent system

$$
\left\{\begin{aligned}
& u_{1}^{\prime}(t)= a_{11} u_{1}(t)+a_{12} u_{2}\left(t-\tau_{1}\right)+a_{13} u_{3}\left(t-\tau_{1}\right) \\
& \quad+\sum_{i+j+k \geqslant 2} \frac{1}{i ! j ! k !} f_{i j k}^{(1)} u_{1}^{i}(t) u_{2}^{j}\left(t-\tau_{1}\right) u_{3}^{k}\left(t-\tau_{1}\right), \\
& u_{2}^{\prime}(t)=a_{21} u_{1}\left(t-\tau_{2}\right)+\sum_{i+j \geqslant 2} \frac{1}{i ! j !} f_{i j}^{(2)} u_{1}^{i}\left(t-\tau_{2}\right) u_{2}^{j}(t), \\
& u_{3}^{\prime}(t)=a_{31} u_{1}\left(t-\tau_{2}\right)+a_{33} u_{3}(t)+\sum_{i+j \geqslant 2} \frac{1}{i ! j !} f_{i j}^{(3)} u_{1}^{i}\left(t-\tau_{2}\right) u_{3}^{j}(t),
\end{aligned}\right.
$$

where

$$
\begin{aligned}
& a_{11}=r-\frac{2 r u^{*}}{K}-\frac{a v^{*}}{\left(1+b u^{*}\right)^{2}}-\frac{A w^{*}}{\left(1+B u^{*}\right)^{2}}, \quad a_{12}=-\frac{a u^{*}}{1+b u^{*}}, \\
& a_{13}=-\frac{A u^{*}}{1+B u^{*}}, \quad a_{21}=\frac{e v^{*}}{\left(1+b u^{*}\right)^{2}}, \quad a_{31}=\frac{E w^{*}}{\left(1+b u^{*}\right)^{2}}, \quad a_{33}=-G w^{*}, \\
& f^{(1)}=r u_{1}(t)\left(1-\frac{u_{1}(t)}{K}\right)-\frac{a u_{1}(t) u_{2}\left(t-\tau_{1}\right)}{1+b u_{1}(t)}-\frac{A u_{1}(t) u_{3}\left(t-\tau_{1}\right)}{1+B u_{1}(t)}, \\
& f^{(2)}=u_{2}(t)\left(-d+\frac{e u_{1}\left(t-\tau_{2}\right)}{\left.1+b u_{1}\left(t-\tau_{2}\right)\right)}\right), \\
& f^{(3)}=u_{3}(t)\left(-D-G u_{3}(t)+\frac{E u_{1}\left(t-\tau_{2}\right)}{\left.1+B u_{1}\left(t-\tau_{2}\right)\right)}\right) \\
& f_{i j k}^{(1)}=\left.\frac{\partial^{i+j+k} f^{(1)}}{\partial u_{1}^{i}(t) \partial u_{2}^{j}\left(t-\tau_{1}\right) \partial u_{3}^{k}\left(t-\tau_{1}\right)}\right|_{\left(u^{*}, v^{*}, w^{*}\right)}, \\
& f_{i j}^{(2)}=\left.\frac{\partial^{i+j} f^{(2)}}{\partial u_{1}^{i}\left(t-\tau_{2}\right) \partial u_{2}^{j}(t)}\right|_{\left(u^{*}, v^{*}, w^{*}\right)} ^{(3)}=\left.\frac{\partial^{i+j} f^{(3)}}{\partial u_{1}^{i}\left(t-\tau_{2}\right) \partial u_{3}^{j}(t)}\right|_{\left(u^{*}, v^{*}, w^{*}\right)} .
\end{aligned}
$$

To study the stability of the equilibrium $E^{*}\left(u^{*}, v^{*}, w^{*}\right)$, it is sufficient to study the stability of the origin for the system (2.1). The linearized system of the system (1.2) at origin is

$$
\left\{\begin{array}{l}
u_{1}^{\prime}(t)=a_{11} u_{1}(t)+a_{12} u_{2}\left(t-\tau_{1}\right)+a_{13} u_{3}\left(t-\tau_{1}\right), \\
u_{2}^{\prime}(t)=a_{21} u_{1}\left(t-\tau_{2}\right), \\
u_{3}^{\prime}(t)=a_{31} u_{1}\left(t-\tau_{2}\right)+a_{33} u_{3}(t) .
\end{array}\right.
$$

The characteristic equation resulted from the linear system (2.2) has the form

$$
\lambda^{3}+p_{1} \lambda^{2}+p_{2} \lambda+\left(q_{2} \lambda+q_{3}\right) e^{-\lambda \tau}=0,
$$

where $p_{1}, p_{2}, q_{2}, q_{3}$ are defined by

(2.4) $p_{1}=-\left(a_{11}+a_{33}\right), p_{2}=a_{11} a_{33}, q_{2}=-\left(a_{13} a_{31}+a_{12} a_{21}\right), q_{3}=a_{12} a_{21} a_{33}$ 
and $\tau=\tau_{1}+\tau_{2}$. Next, we will investigate the distribution of roots of Eq.(2.3). When $\tau=0,(2.3)$ is reduced to the following third degree algebraic equation

$$
\lambda^{3}+p_{1} \lambda^{2}+\left(p_{2}+q_{2}\right) \lambda+q_{3}=0 .
$$

In order to determine the distribution of roots of (2.5), we first give the following lemma.

Lemma 2.1. Let the assumption (H1) hold and the components $u^{*}, v^{*}$ and $w^{*}$ of the positive equilibrium $E^{*}\left(u^{*}, v^{*}, w^{*}\right)$ of system (1.2) satisfy the following condition

$$
r-\frac{2 r u^{*}}{K}-\frac{a v^{*}}{\left(1+b u^{*}\right)^{2}}-\frac{A w^{*}}{\left(1+B u^{*}\right)^{2}}<0 .
$$

In addition, $p_{1}, p_{2}, q_{2}, q_{3}$ are defined by (2.4). Then $p_{1}>0$ and $p_{1}\left(p_{2}+q_{2}\right)>q_{3}$.

Proof. The assumption (H2) means that $a_{11}<0$ and hence $p_{1}=-\left(a_{11}+a_{33}\right)>$ 0 because $a_{33}=-G w^{*}<0$. In addition, we have from the definitions of $p_{1}, p_{2}, q_{2}$ and $q_{3}$ that

$$
p_{1}\left(p_{2}+q_{2}\right)-q_{3}=-a_{11}^{2} a_{33}+a_{11} a_{13} a_{31}+a_{11} a_{12} a_{21}-a_{11} a_{33}^{2}+a_{13} a_{31} a_{33} .
$$

Notice that $a_{11}<0, a_{12} a_{21}<0, a_{13} a_{31}<0$ and $a_{33}<0$ under the hypotheses (H1) and (H2). Therefore, $p_{1}\left(p_{2}+q_{2}\right)-q_{3}>0$ and the proof is complete.

It follows from Lemma 2.1 and the Routh-Hurwitz criteria that, under the hypotheses (H1) and (H2), all roots of (2.5) have negative real parts and thus the zero equilibrium of system (2.1) is asymptotically stable when $\tau=0$. Now for $\tau>0$, let $\lambda=i \omega(\omega>0)$ be the root of Eq.(2.3). Then we have

$$
-i \omega^{3}-p_{1} \omega^{2}+i \omega p_{2}+\left(i w q_{2}+q_{3}\right)(\cos \omega \tau-i \sin \omega \tau)=0 .
$$

Separating the real and imaginary parts yields

$$
\left\{\begin{array}{l}
q_{3} \cos \omega \tau+q_{2} \omega \sin \omega \tau-p_{1} \omega^{2}=0, \\
q_{2} \omega \cos \omega \tau-q_{3} \sin \omega \tau+p_{2} \omega-\omega^{3}=0,
\end{array}\right.
$$

which imply that

$$
\omega^{6}+\left(p_{1}^{2}-2 p_{2}\right) \omega^{4}+\left(p_{2}^{2}-q_{2}^{2}\right) \omega^{2}-q_{3}^{2}=0 .
$$

If the equation (2.8) has a positive root $\omega_{0}$, then from $(2.7)$ the corresponding values of $\tau$ such that (2.3) has the purely imaginary root $i \omega_{0}$ can be denoted as

$$
\tau_{k}=\frac{1}{\omega_{0}} \arccos \left(\frac{q_{2} \omega_{0}^{4}+p_{1} q_{3} \omega_{0}^{2}-p_{2} q_{2} \omega_{0}^{2}}{q_{2}^{2} \omega_{0}^{2}+q_{3}^{2}}\right)+\frac{2 k \pi}{\omega_{0}}, k=0,1,2, \ldots
$$

For (2.8), we first have the following result.

Lemma 2.2. Suppose that the condition (H1) holds. Then (2.8) has at least one positive real root. 
Proof. Introducing the new parameter $z$ and assuming $z=\omega^{2}$. Then (2.8) can be denoted simply as the following equation

$$
z^{3}+\left(p_{1}^{2}-2 p_{2}\right) z^{2}+\left(p_{2}^{2}-q_{2}^{2}\right) z-q_{3}^{2}=0 .
$$

Let

$$
g(z)=z^{3}+\left(p_{1}^{2}-2 p_{2}\right) z^{2}+\left(p_{2}^{2}-q_{2}^{2}\right) z-q_{3}^{2} .
$$

It is easy to see that $\lim _{z \rightarrow+\infty} g(z)=+\infty$ and $g(0)=-q_{3}^{2}<0$. Hence, there exists at least a $z_{0} \in(0,+\infty)$ such that $g\left(z_{0}\right)=0$. This completes the proof.

Lemma 2.3. Assume that $g^{\prime}\left(\omega_{0}^{2}\right) \neq 0$. Then the transversality conditions $d \operatorname{Re} \lambda(\tau) /\left.d \tau\right|_{\tau=\tau_{k}} \neq 0(k=0,1,2, \ldots)$ hold.

Proof. Letting $\lambda(\tau)$ be the root of (2.3) and differentiating two sides of (2.3) with respect to $\tau$, one can obtain

$$
\left[3 \lambda^{2}+2 p_{1} \lambda+p_{2}+q_{2} e^{-\lambda \tau}-\tau\left(q_{2} \lambda+q_{3}\right) e^{-\lambda \tau}\right] \frac{d \lambda}{d \tau}=\lambda\left(q_{2} \lambda+q_{3}\right) e^{-\lambda \tau} .
$$

It follows easily from (2.3) that

$$
\left(q_{2} \lambda+q_{3}\right) e^{-\lambda \tau}=-\lambda\left(\lambda^{2}+p_{1} \lambda+p_{2}\right) .
$$

Thus one can from (2.10) obtain

$$
\left(\frac{d \lambda}{d \tau}\right)^{-1}=-\frac{3 \lambda^{2}+2 p_{1} \lambda+p_{2}}{\lambda^{2}\left(\lambda^{2}+p_{1} \lambda+p_{2}\right)}+\frac{q_{2}}{\lambda\left(q_{2} \lambda+q_{3}\right)}-\frac{\tau}{\lambda} .
$$

It follows from the fact $\lambda\left(\tau_{k}\right)=i \omega_{0}$ that

$$
\begin{aligned}
\operatorname{Re}\left[\left.\left(\frac{d \lambda}{d \tau}\right)^{-1}\right|_{\tau=\tau_{k}}\right] & =\operatorname{Re}\left(\left.\frac{3 \lambda^{2}+2 p_{1} \lambda+p_{2}}{\lambda^{2}\left(\lambda^{2}+p_{1} \lambda+p_{2}\right)}\right|_{\tau=\tau_{k}}\right)+\operatorname{Re}\left(\left.\frac{q_{2}}{\lambda\left(q_{2} \lambda+q_{3}\right)}\right|_{\tau=\tau_{k}}\right) \\
& =-\operatorname{Re}\left(\frac{p_{2}-3 \omega_{0}^{2}+2 i p_{1} \omega_{0}}{\omega_{0}^{2}\left(p_{2}-\omega_{0}^{2}+i p_{1} \omega_{0}\right)}\right)+\operatorname{Re}\left(\frac{q_{2}}{-q_{2} \omega_{0}^{2}+i q_{3} \omega_{0}}\right) \\
& =\frac{\left(p_{2}-3 \omega_{0}^{2}\right)\left(p_{2}-\omega_{0}^{2}\right)+2 p_{1}^{2} \omega_{0}^{2}}{\omega_{0}^{2}\left[\left(p_{2}-\omega_{0}^{2}\right)^{2}+p_{1}^{2} \omega_{0}^{2}\right]}-\frac{q_{2}^{2}}{q_{3}^{2}+\left(q_{2} \omega_{0}\right)^{2}} .
\end{aligned}
$$

Notice that (2.3) has the purely imaginary root $i \omega_{0}$ when $\tau=\tau_{k}$, that is,

$$
-i \omega_{0}^{3}-p_{1} \omega_{0}^{2}+i p_{2} \omega_{0}+\left(i q_{2} \omega_{0}+q_{3}\right) e^{-i \omega_{0} \tau}=0,
$$

which implies that

$$
\left|-p_{1} \omega_{0}^{2}+i\left(p_{2} \omega_{0}-\omega_{0}^{3}\right)\right|=\left|q_{3}+i q_{2} \omega_{0}\right|,
$$

that is,

$$
\omega_{0}^{2}\left[\left(p_{2}-\omega_{0}^{2}\right)^{2}+p_{1}^{2} \omega_{0}^{2}\right]=q_{3}^{2}+\left(q_{2} \omega_{0}\right)^{2} .
$$


(2.11) and (2.12) give

$$
\begin{aligned}
\operatorname{Re}\left[\left.\left(\frac{d \lambda}{d \tau}\right)^{-1}\right|_{\tau=\tau_{k}}\right] & =\frac{3 \omega_{0}^{4}+2\left(p_{1}^{2}-2 p_{2}\right) \omega_{0}^{2}+p_{2}^{2}-q_{2}^{2}}{\omega_{0}^{2}\left[\left(p_{2}-\omega_{0}^{2}\right)^{2}+p_{1}^{2} \omega_{0}^{2}\right]} \\
& =\frac{g^{\prime}\left(\omega_{0}^{2}\right)}{\omega_{0}^{2}\left[\left(p_{2}-\omega_{0}^{2}\right)^{2}+p_{1}^{2} \omega_{0}^{2}\right]} \neq 0 .
\end{aligned}
$$

This implies $\left.\frac{d \operatorname{Re} \lambda}{d \tau}\right|_{\tau=\tau_{k}} \neq 0$ and hence the proof is complete.

Combining Lemma 2.3 and the Hopf bifurcation theorem for functional differential equations [6], we can state the following theorem.

Theorem 2.4. Suppose that conditions (H1) and $(\mathrm{H} 2)$ hold and $g^{\prime}\left(\omega_{0}^{2}\right) \neq 0$. Then the positive equilibrium $E^{*}\left(u^{*}, v^{*}, w^{*}\right)$ is asymptotically stable for all $0 \leqslant$ $\tau<\tau_{0}$. Furthermore, the system (1.2) undergoes a Hopf bifurcation at the positive equilibrium $E^{*}\left(u^{*}, v^{*}, w^{*}\right)$ when $\tau=\tau_{k}(k=0,1,2, \ldots)$.

\section{Direction of Hopf bifurcations and stability of the bifurcating periodic orbits}

At this time, the stability of positive equilibrium $E^{*}\left(u^{*}, v^{*}, w^{*}\right)$ of the system (1.2) and the existence of Hopf bifurcations at $E^{*}\left(u^{*}, v^{*}, w^{*}\right)$ have been obtained. In this section, we will investigate further the properties of Hopf bifurcations obtained by Theorem 2.4 and the stability of bifurcating periodic solutions occurring through Hopf bifurcations by using the normal form theory and the center manifold reduction for the retarded functional differential equations due to Hassard, Kazarinoff and Wan [7].

Now, we assume that the conditions of Theorem 2.4 hold and consider the equivalent system (2.1) of the system $(1.2)$ in the phase space $C=C\left([-\tau, 0], \mathbb{R}^{3}\right)$. Let $u(t)=\left(u_{1}(t), u_{2}(t), u_{3}(t)\right)^{T}, u_{t}(\theta)=u(t+\theta), \theta \in[-\tau, 0]$ for $\varphi \in C$, and $L(\tau)\left(u_{t}\right), f\left(u_{t}, \tau\right)$ are given, respectively, by

$$
L(\tau) \varphi(\theta)=A \varphi(0)+B_{1} \varphi\left(-\tau_{1}\right)+B_{2} \varphi\left(-\tau_{2}\right),
$$

and

$$
f(\varphi, \tau)=\left(\begin{array}{c}
\sum_{i+j+k \geqslant 2} \frac{1}{i ! j ! k !} f_{i j k}^{(1)} \varphi_{1}^{i}(0) \varphi_{2}^{j}\left(-\tau_{1}\right) \varphi_{3}^{k}\left(-\tau_{1}\right) \\
\sum_{i+j \geqslant 2} \frac{1}{i ! j !} f_{i j}^{(2)} \varphi_{1}^{i}\left(-\tau_{2}\right) \varphi_{2}^{j}(0) \\
\sum_{i+j \geqslant 2} \frac{1}{i ! j !} f_{i j}^{(3)} \varphi_{1}^{i}\left(-\tau_{2}\right) \varphi_{3}^{j}(0)
\end{array}\right)
$$

where

$$
A=\left(\begin{array}{ccc}
a_{11} & 0 & 0 \\
0 & 0 & 0 \\
0 & 0 & a_{33}
\end{array}\right), \quad B_{1}=\left(\begin{array}{ccc}
0 & a_{12} & a_{13} \\
0 & 0 & 0 \\
0 & 0 & 0
\end{array}\right), \quad B_{2}=\left(\begin{array}{ccc}
0 & 0 & 0 \\
a_{21} & 0 & 0 \\
a_{31} & 0 & 0
\end{array}\right) .
$$


Then the system $(2.1)$ can be rewritten into the following vector form

$$
u^{\prime}(t)=L(\tau)\left(u_{t}\right)+f\left(u_{t}, \tau\right) .
$$

By the Riesz representation theorem, there exists a $3 \times 3$ matrix function $\eta(\theta, \tau),-\tau \leqslant \theta \leqslant 0$, whose elements are of bounded variation such that

$$
L(\varphi)=\int_{-\tau}^{0} d \eta(\theta, \tau) \varphi(\theta) \quad \text { for } \varphi \in C .
$$

In fact, we can choose

$$
\eta(\theta, \tau)=A \delta(\theta)-B_{1} \delta\left(\theta+\tau_{1}\right)-B_{2} \delta\left(\theta+\tau_{2}\right),
$$

where $\delta$ denote Dirac delta function. For $\varphi \in C^{1}\left([-\tau, 0], \mathbb{R}^{3}\right)$, define

$$
A(\tau) \varphi=\left\{\begin{array}{l}
\frac{d \varphi(\theta)}{d \theta}, \theta \in[-\tau, 0] \\
\int_{-\tau}^{0} d \eta(s, \tau) \varphi(s), \theta=0
\end{array}\right.
$$

and

$$
R(\tau) \varphi=\left\{\begin{array}{l}
0, \theta \in[-\tau, 0) \\
f(\varphi, \tau), \theta=0
\end{array}\right.
$$

Then the system (3.2) can be written as the following abstract operator differential equation

$$
u_{t}^{\prime}=A(\tau) u_{t}+R(\tau) u_{t} .
$$

For fixed $k \in\{0,1, \ldots\}$, denote $\tau_{1}$ and $\tau_{2}$ by $\tau_{1 k}$ and $\tau_{2 k}$ such that $\tau_{1}+\tau_{2}=\tau_{k}$. Let $\psi \in C^{1}\left(\left[0, \tau_{k}\right],\left(\mathbb{R}^{3}\right)^{*}\right)$. Then the adjoint operator $A^{*}$ of $A\left(\tau_{k}\right)$ is defined as

$$
A^{*} \psi(s)=\left\{\begin{array}{l}
-\frac{d \psi(s)}{d s}, s \in\left(0, \tau_{k}\right] \\
\int_{-\tau_{k}}^{0} d \eta^{T}\left(t, \tau_{k}\right) \psi(-t), s=0
\end{array}\right.
$$

For $\psi \in C^{1}\left(\left[0, \tau_{k}\right],\left(\mathbb{R}^{3}\right)^{*}\right)$ and $\varphi \in C^{1}\left(\left[-\tau_{k}, 0\right], \mathbb{R}^{3}\right)$, define a bilinear inner product

$$
\langle\psi(s), \varphi(\theta)\rangle=\bar{\psi}(0) \varphi(0)-\int_{-\tau_{k}}^{0} \int_{\xi=0}^{\theta} \bar{\psi}(\xi-\theta) d \eta(\theta) \varphi(\xi) d \xi,
$$

where $\eta(\theta)=\eta\left(\theta, \tau_{k}\right)$. From the discussions in Section 2, we know that $A\left(\tau_{k}\right)$ has a pair of purely imaginary eigenvalues $\pm i \omega_{0}$. Then $\pm i \omega_{0}$ are also eigenvalues of $A^{*}$.

From the definition of $A\left(\tau_{k}\right)$, we know that the eigenfunction of $A\left(\tau_{k}\right)$ corresponding to the eigenvalue $i \omega_{0}$ has the form $q(\theta)=q(0) e^{i \omega_{0} \theta},-\tau_{k} \leqslant \theta \leqslant 0$. In addition, $q(0)$ satisfies the following equation

$$
\left(\begin{array}{ccc}
a_{11}-i \omega_{0} & a_{12} e^{-i \omega_{0} \tau_{1 k}} & a_{13} e^{-i \omega_{0} \tau_{1 k}} \\
a_{21} e^{-i \omega_{0} \tau_{2 k}} & -i \omega_{0} & 0 \\
a_{31} e^{-i \omega_{0} \tau_{2 k}} & 0 & a_{33}-i \omega_{0}
\end{array}\right) q(0)=\left(\begin{array}{l}
0 \\
0 \\
0
\end{array}\right)
$$


One can easily obtain

$$
q(0)=(1, \alpha, \beta)^{T}
$$

where

$$
\alpha=\frac{a_{21} e^{-i \omega_{0} \tau_{2 k}}}{i \omega_{0}}, \quad \beta=\frac{a_{31} e^{-i \omega_{0} \tau_{2 k}}}{i \omega_{0}-a_{33}} .
$$

Similarly, let $q^{*}(s)=D\left(1, \alpha^{*}, \beta^{*}\right) e^{i \omega_{0} s}$ is the eigenfunction of $A^{*}$ corresponding to $-i \omega_{0}$ and we can get

$$
\alpha^{*}=-\frac{a_{12} e^{-i \omega_{0} \tau_{1 k}}}{i \omega_{0}}, \quad \beta^{*}=-\frac{a_{13} e^{-i \omega_{0} \tau_{1 k}}}{i \omega_{0}+a_{33}} .
$$

In order to assure $\left\langle q^{*}(s), q(\theta)\right\rangle=1$, we need to determine the value of $D$. Combining the above definitions, we have

$$
\begin{aligned}
& \left\langle q^{*}(s), q(\theta)\right\rangle \\
= & \overline{q^{*}}(0) q(0)-\int_{-\tau}^{0} \int_{\xi=0}^{\theta} \overline{q^{*}}(\xi-\theta) d \eta(\theta) q(\xi) d \xi \\
= & \bar{D}\left(1, \overline{\alpha^{*}}, \overline{\beta^{*}}\right)(1, \alpha, \beta)^{T}-\int_{-\tau}^{0} \int_{\xi=0}^{\theta} \bar{D}\left(1, \overline{\alpha^{*}}, \overline{\beta^{*}}\right) e^{-i \omega_{0}(\xi-\theta)} d \eta(\theta)(1, \alpha, \beta)^{T} e^{i \omega_{0} \xi} d \xi \\
= & \bar{D}\left[1+\alpha \overline{\alpha^{*}}+\beta \overline{\beta^{*}}-\int_{-\tau}^{0}\left(1, \overline{\alpha^{*}}, \overline{\beta^{*}}\right) \theta e^{i \omega_{0} \theta} d \eta(\theta)(1, \alpha, \beta)^{T}\right] \\
= & \bar{D}\left[1+\alpha \overline{\alpha^{*}}+\beta \overline{\beta^{*}}+\tau_{2 k} e^{-i \omega_{0} \tau_{2 k}}\left(a_{21} \alpha^{*}+a_{31} \beta^{*}\right)+\tau_{1 k} e^{-i \omega_{0} \tau_{1 k}}\left(a_{12} \alpha+a_{13} \beta\right)\right] .
\end{aligned}
$$

Thus, we can choose

$$
D=\left[1+\bar{\alpha} \alpha^{*}+\bar{\beta} \beta^{*}+\tau_{2 k} e^{i \omega_{0} \tau_{2 k}}\left(a_{21} \overline{\alpha^{*}}+a_{31} \overline{\beta^{*}}\right)+\tau_{1 k} e^{i \omega_{0} \tau_{1 k}}\left(a_{12} \bar{\alpha}+a_{13} \bar{\beta}\right)\right]^{-1},
$$

such that

$$
\left\langle q^{*}(s), q(\theta)\right\rangle=1, \quad\left\langle q^{*}(s), \bar{q}(\theta)\right\rangle=0 .
$$

Let $u_{t}$ be the solution of (3.6) and define

$$
z(t)=\left\langle q^{*}(s), u_{t}(\theta)\right\rangle
$$

and

$$
W(t, \theta)=u_{t}(\theta)-2 \operatorname{Re}\{z(t) q(\theta)\}
$$

From [7], we have

$$
W(t, \theta)=W(z(t), \bar{z}(t), \theta)
$$

where

(3.10) $W(z, \bar{z}, \theta)=W_{20}(\theta) \frac{z^{2}}{2}+W_{11}(\theta) z \bar{z}+W_{02}(\theta) \frac{\bar{z}^{2}}{2}+W_{30}(\theta) \frac{z^{3}}{6}+\cdots$, $z$ and $\bar{z}$ are local coordinates for center manifold $C_{0}$ in the direction of $q$ and $q^{*}$. Note that $W$ is real if $y_{t}$ is real and therefore we only consider real solution. 
Notice $u_{t}^{\prime}=A\left(\tau_{k}\right) u_{t}+R\left(\tau_{k}\right) u_{t}$ when $\tau=\tau_{k}$. From the definition of $R(\tau)$ and (3.8), we have

$$
\begin{aligned}
z^{\prime}(t) & =\left\langle q^{*}(s), u_{t}^{\prime}\right\rangle=\left\langle q^{*}(s), A\left(\tau_{k}\right) u_{t}+R\left(\tau_{k}\right) u_{t}\right\rangle \\
& =\left\langle q^{*}(s), A\left(\tau_{k}\right) u_{t}\right\rangle+\left\langle q^{*}(s), R\left(\tau_{k}\right) u_{t}\right\rangle \\
& =\left\langle-i \omega_{0} q^{*}(s), u_{t}\right\rangle+\bar{q}^{*}(0) f\left(u_{t}(\theta), \tau_{k}\right) \\
& =i \omega_{0} z(t)+\bar{q}^{*}(0) f_{0}(z(t), \bar{z}(t)) .
\end{aligned}
$$

Rewrite (3.11) as

$$
z^{\prime}(t)=i \omega_{0} z(t)+g(z(t), \bar{z}(t))
$$

where

$$
g(z, \bar{z})=g_{20} \frac{z^{2}}{2}+g_{11} z \bar{z}+g_{02} \frac{\bar{z}^{2}}{2}+g_{21} \frac{z^{2} \bar{z}}{2}+\cdots .
$$

From (3.9), one can get

$$
\begin{aligned}
u_{t}(\theta)= & W(t, \theta)+2 \operatorname{Re}\{z(t) q(\theta)\} \\
= & W(t, \theta)+z(t) q(\theta)+\bar{z}(t) \bar{q}(\theta) \\
= & W_{20}(\theta) \frac{z^{2}}{2}+W_{11}(\theta) z \bar{z}+W_{02}(\theta) \frac{\bar{z}^{2}}{2} \\
& +(1, \alpha, \beta)^{\mathrm{T}} e^{i \omega_{0} \theta} z+(1, \bar{\alpha}, \bar{\beta})^{\mathrm{T}} e^{-i \omega_{0} \theta} \bar{z}+\cdots .
\end{aligned}
$$

Therefore,

$$
\begin{aligned}
& u_{t}^{(1)}(0)=W_{20}^{(1)}(0) \frac{z^{2}}{2}+W_{11}^{(1)}(0) z \bar{z}+W_{02}^{(1)}(0) \frac{\bar{z}^{2}}{2}+z+\bar{z}+\cdots, \\
& u_{t}^{(1)}\left(-\tau_{2 k}\right)=W_{20}^{(1)}\left(-\tau_{2 k}\right) \frac{z^{2}}{2}+W_{11}^{(1)}\left(-\tau_{2 k}\right) z \bar{z}+W_{02}^{(1)}(0) \frac{\bar{z}^{2}}{2} \\
& +e^{-i \omega_{0} \tau_{2 k}} z+e^{i \omega_{0} \tau_{2 k}} \bar{z}+\cdots, \\
& u_{t}^{(2)}(0)=W_{20}^{(2)}(0) \frac{z^{2}}{2}+W_{11}^{(2)}(0) z \bar{z}+W_{02}^{(2)}(0) \frac{\bar{z}^{2}}{2} \\
& +\alpha z+\overline{\alpha z}+\cdots, \\
& u_{t}^{(2)}\left(-\tau_{1 k}\right)=W_{20}^{(2)}\left(-\tau_{1 k}\right) \frac{z^{2}}{2}+W_{11}^{(2)}\left(-\tau_{1 k}\right) z \bar{z}+W_{02}^{(2)}\left(-\tau_{1 k}\right) \frac{\bar{z}^{2}}{2} \\
& +\alpha e^{-i \omega_{0} \tau_{1 k}} z+\bar{\alpha} e^{i \omega_{0} \tau_{1 k}} \bar{z}+\cdots, \\
& u_{t}^{(3)}(0)=W_{20}^{(3)}(0) \frac{z^{2}}{2}+W_{11}^{(3)}(0) z \bar{z}+W_{02}^{(3)}(0) \frac{\bar{z}^{2}}{2}+\beta z+\overline{\beta z}+\cdots, \\
& u_{t}^{(3)}\left(-\tau_{1 k}\right)=W_{20}^{(3)}\left(-\tau_{1 k}\right) \frac{z^{2}}{2}+W_{11}^{(3)}\left(-\tau_{1 k}\right) z \bar{z}+W_{02}^{(3)}\left(-\tau_{1 k}\right) \frac{\bar{z}^{2}}{2} \\
& +\beta e^{-i \omega_{0} \tau_{1 k}} z+\bar{\beta} e^{i \omega_{0} \tau_{1 k}} \bar{z}+\cdots .
\end{aligned}
$$


From the definition of $f^{(1)}$, one can observe easily that $f_{020}^{(1)}=f_{002}^{(1)}=0$. Therefore, we have from (3.1) that

$$
f_{0}(z(t), \bar{z}(t))=\left(f_{0}^{1}(z(t), \bar{z}(t)), f_{0}^{2}(z(t), \bar{z}(t)), f_{0}^{3}(z(t), \bar{z}(t))\right)^{\mathrm{T}},
$$

where

$$
\begin{aligned}
& f_{0}^{1}(z(t), \bar{z}(t)) \\
= & \left(f_{200}^{(1)} e^{i \omega_{0} \tau_{1 k}}+2 \alpha f_{110}^{(1)}+2 \beta f_{101}^{(1)}+2 \alpha \beta f_{011}^{(1)} e^{-i \omega_{0} \tau_{1 k}}\right) e^{-i \omega_{0} \tau_{1 k}} \frac{z^{2}}{2} \\
& +\left(f_{200}^{(1)}+2 f_{110}^{(1)} \operatorname{Re}\left\{\alpha e^{-i \omega_{0} \tau_{1 k}}\right\}+2 f_{101}^{(1)} \operatorname{Re}\left\{\beta e^{-i \omega_{0} \tau_{1 k}}\right\}+2 f_{011}^{(1)} \operatorname{Re}\{\alpha \bar{\beta}\}\right) z \bar{z} \\
& +\left(f_{200}^{(1)} e^{-i \omega_{0} \tau_{1 k}}+2 \bar{\alpha} f_{110}^{(1)}+2 \bar{\beta} f_{101}^{(1)}+2 \bar{\alpha} \bar{\beta} f_{011}^{(1)} e^{i \omega_{0} \tau_{1 k}}\right) e^{i \omega_{0} \tau_{1 k}} \frac{\bar{z}^{2}}{2} \\
& +\left\{f_{200}^{(1)}\left[W_{20}^{(1)}(0)+2 W_{11}^{(1)}(0)\right]\right. \\
& +f_{110}^{(1)}\left[W_{20}^{(2)}\left(-\tau_{1 k}\right)+2 W_{11}^{(2)}\left(-\tau_{1 k}\right)+\bar{\alpha} e^{i \omega_{0} \tau_{1 k}} W_{20}^{(1)}(0)+2 \alpha e^{-i \omega_{0} \tau_{1 k}} W_{11}^{(1)}(0)\right] \\
& +f_{101}^{(1)}\left[W_{20}^{(3)}\left(-\tau_{1 k}\right)+2 W_{11}^{(3)}\left(-\tau_{1 k}\right)+\bar{\beta} e^{i \omega_{0} \tau_{1 k}} W_{20}^{(1)}(0)+2 \beta e^{-i \omega_{0} \tau_{1 k}} W_{11}^{(1)}(0)\right] \\
+ & f_{011}^{(1)}\left[\bar{\beta} e^{i \omega_{0} \tau_{1 k}} W_{20}^{(2)}\left(-\tau_{1 k}\right)+2 \beta e^{-i \omega_{0} \tau_{1 k}} W_{11}^{(2)}\left(-\tau_{1 k}\right)+\bar{\alpha} e^{i \omega_{0} \tau_{1 k}} W_{20}^{(3)}\left(-\tau_{1 k}\right)\right. \\
& \left.\left.+2 \alpha e^{-i \omega_{0} \tau_{1 k}} W_{11}^{(3)}\left(-\tau_{1 k}\right)\right]\right\} \frac{z^{2} \bar{z}}{2}+\cdots \\
+ & f_{0}^{2}(z(t), \bar{z}(t)) \\
= & \left(f_{20}^{(2)} e^{-2 i \omega_{0} \tau_{2 k}}+f_{02}^{(2)} \alpha^{2}+2 \alpha f_{11}^{(2)} e^{-i \omega_{0} \tau_{2 k}}\right) \frac{z^{2}}{2} \\
+ & \left(f_{20}^{(2)}+f_{11}^{(2)}\left(\bar{\alpha} e^{-i \omega_{0} \tau_{2 k}}+\alpha e^{i \omega_{0} \tau_{2 k}}\right)+f_{20}^{(2)} \alpha \bar{\alpha}\right) z \bar{z} \\
+ & \left(f_{20}^{(2)} e^{2 i \omega_{0} \tau_{2 k}}+f_{02}^{(2)} \bar{\alpha}^{2}+2 \bar{\alpha} f_{11}^{(2)} e^{i \omega_{0} \tau_{2 k}}\right) \frac{\bar{z}^{2}}{2} \\
+ & \left\{f_{20}^{(2)}\left[e^{i \omega_{0} \tau_{2 k}} W_{20}^{(1)}\left(-\tau_{2 k}\right)+2 e^{-i \omega_{0} \tau_{2 k}} W_{11}^{(1)}\left(-\tau_{2 k}\right)+2 \alpha W_{11}^{(1)}\left(-\tau_{2 k}\right)+e^{i \omega_{0} \tau_{2 k}} W_{20}^{(2)}(0)+2 e^{-i \omega_{0} \tau_{2 k}} W_{11}^{(2)}(0)\right]\right. \\
& \left.\left.+2 \alpha W_{11}^{(2)}(0)\right]\right\} \frac{z^{2} \bar{z}}{2}+\cdots \\
+ & (0) \\
+ &
\end{aligned}
$$

and

$$
\begin{aligned}
& f_{0}^{3}(z(t), \bar{z}(t)) \\
= & \left(f_{20}^{(3)} e^{-2 i \omega_{0} \tau_{2 k}}+f_{02}^{(3)} \beta^{2}+2 \beta f_{11}^{(3)} e^{-i \omega_{0} \tau_{2 k}}\right) \frac{z^{2}}{2} \\
& +\left(f_{20}^{(3)}+f_{11}^{(3)}\left(\bar{\beta} e^{-i \omega_{0} \tau_{2 k}}+\beta e^{i \omega_{0} \tau_{2 k}}\right)+f_{20}^{(3)} \beta \bar{\beta}\right) z \bar{z}
\end{aligned}
$$




$$
\begin{aligned}
& +\left(f_{20}^{(3)} e^{2 i \omega_{0} \tau_{2 k}}+f_{02}^{(3)} \bar{\beta}^{2}+2 \bar{\beta} f_{11}^{(3)} e^{i \omega_{0} \tau_{2 k}}\right) \frac{\bar{z}^{2}}{2} \\
& +\left\{f_{20}^{(3)}\left[e^{i \omega_{0} \tau_{2 k}} W_{20}^{(1)}\left(-\tau_{2 k}\right)+2 e^{-i \omega_{0} \tau_{2 k}} W_{11}^{(1)}\left(-\tau_{2 k}\right)\right]\right. \\
& +f_{11}^{(3)}\left[\bar{\beta} W_{20}^{(1)}\left(-\tau_{2 k}\right)+2 \beta W_{11}^{(1)}\left(-\tau_{2 k}\right)+e^{i \omega_{0} \tau_{2 k}} W_{20}^{(3)}(0)+2 e^{-i \omega_{0} \tau_{2 k}} W_{11}^{(3)}(0)\right] \\
& \left.+f_{20}^{(3)}\left[\bar{\beta} W_{20}^{(3)}(0)+2 \beta W_{11}^{(3)}(0)\right]\right\} \frac{z^{2} \bar{z}}{2}+\cdots
\end{aligned}
$$

Thus one can obtain

$$
\begin{aligned}
& g(z, \bar{z}) \\
& =g_{20} \frac{z^{2}}{2}+g_{11} z \bar{z}+g_{02} \frac{\bar{z}^{2}}{2}+g_{21} \frac{z^{2} \bar{z}}{2}+\cdots \\
& =\bar{q}^{*}(0) f_{0}(z(t), \bar{z}(t)) \\
& =\bar{D}\left[\left(f_{200}^{(1)} e^{i \omega_{0} \tau_{1 k}}+2 \alpha f_{110}^{(1)}+2 \beta f_{101}^{(1)}+2 \alpha \beta f_{011}^{(1)} e^{-i \omega_{0} \tau_{1 k}}\right) e^{-i \omega_{0} \tau_{1 k}}\right. \\
& +\overline{\alpha^{*}}\left(f_{20}^{(2)} e^{-2 i \omega_{0} \tau_{2 k}}+f_{02}^{(2)} \alpha^{2}+2 \alpha f_{11}^{(2)} e^{-i \omega_{0} \tau_{2 k}}\right) \\
& \left.+\overline{\beta^{*}}\left(f_{20}^{(3)} e^{-2 i \omega_{0} \tau_{2 k}}+f_{02}^{(3)} \beta^{2}+2 \beta f_{11}^{(3)} e^{-i \omega_{0} \tau_{2 k}}\right)\right] \frac{z^{2}}{2} \\
& +\bar{D}\left[f_{200}^{(1)}+2 f_{110}^{(1)} \operatorname{Re}\left\{\alpha e^{-i \omega_{0} \tau_{1 k}}\right\}+2 f_{101}^{(1)} \operatorname{Re}\left\{\beta e^{-i \omega_{0} \tau_{1 k}}\right\}+2 f_{011}^{(1)} \operatorname{Re}\{\alpha \bar{\beta}\}\right. \\
& +\overline{\alpha^{*}}\left(f_{20}^{(2)}+f_{11}^{(2)}\left(\bar{\alpha} e^{-i \omega_{0} \tau_{2 k}}+\alpha e^{i \omega_{0} \tau_{2 k}}\right)+f_{20}^{(2)} \alpha \bar{\alpha}\right) \\
& \left.+\overline{\beta^{*}}\left(f_{20}^{(3)}+f_{11}^{(3)}\left(\bar{\beta} e^{-i \omega_{0} \tau_{2 k}}+\beta e^{i \omega_{0} \tau_{2 k}}\right)+f_{20}^{(3)} \beta \bar{\beta}\right)\right] z \bar{z} \\
& +\bar{D}\left[\left(f_{200}^{(1)} e^{-i \omega_{0} \tau_{1 k}}+2 \bar{\alpha} f_{110}^{(1)}+2 \bar{\beta} f_{101}^{(1)}+2 \bar{\alpha} \bar{\beta} f_{011}^{(1)} e^{i \omega_{0} \tau_{1 k}}\right) e^{i \omega_{0} \tau_{1 k}}\right. \\
& +\overline{\alpha^{*}}\left(f_{20}^{(2)} e^{2 i \omega_{0} \tau_{2 k}}+f_{02}^{(2)} \bar{\alpha}^{2}+2 \bar{\alpha} f_{11}^{(2)} e^{i \omega_{0} \tau_{2 k}}\right) \\
& \left.+\overline{\beta^{*}}\left(f_{20}^{(3)} e^{2 i \omega_{0} \tau_{2 k}}+f_{02}^{(3)} \bar{\beta}^{2}+2 \bar{\beta} f_{11}^{(3)} e^{i \omega_{0} \tau_{2 k}}\right)\right] \frac{\bar{z}^{2}}{2} \\
& +\bar{D}\left\{f_{200}^{(1)}\left[W_{20}^{(1)}(0)+2 W_{11}^{(1)}(0)\right]\right. \\
& +f_{110}^{(1)}\left[W_{20}^{(2)}\left(-\tau_{1 k}\right)+2 W_{11}^{(2)}\left(-\tau_{1 k}\right)+\bar{\alpha} e^{i \omega_{0} \tau_{1 k}} W_{20}^{(1)}(0)+2 \alpha e^{-i \omega_{0} \tau_{1 k}} W_{11}^{(1)}(0)\right] \\
& +f_{101}^{(1)}\left[W_{20}^{(3)}\left(-\tau_{1 k}\right)+2 W_{11}^{(3)}\left(-\tau_{1 k}\right)+\bar{\beta} e^{i \omega_{0} \tau_{1 k}} W_{20}^{(1)}(0)+2 \beta e^{-i \omega_{0} \tau_{1 k}} W_{11}^{(1)}(0)\right] \\
& +f_{011}^{(1)}\left[\bar{\beta} e^{i \omega_{0} \tau_{1 k}} W_{20}^{(2)}\left(-\tau_{1 k}\right)+2 \beta e^{-i \omega_{0} \tau_{1 k}} W_{11}^{(2)}\left(-\tau_{1 k}\right)+\bar{\alpha} e^{i \omega_{0} \tau_{1 k}} W_{20}^{(3)}\left(-\tau_{1 k}\right)\right. \\
& \left.+2 \alpha e^{-i \omega_{0} \tau_{1 k}} W_{11}^{(3)}\left(-\tau_{1 k}\right)\right] \\
& +\overline{\alpha^{*}}\left[f_{20}^{(2)}\left(e^{i \omega_{0} \tau_{2 k}} W_{20}^{(1)}\left(-\tau_{2 k}\right)+2 e^{-i \omega_{0} \tau_{2 k}} W_{11}^{(1)}\left(-\tau_{2 k}\right)\right)\right.
\end{aligned}
$$




$$
\begin{aligned}
& +f_{11}^{(2)}\left(\bar{\alpha} W_{20}^{(1)}\left(-\tau_{2 k}\right)+2 \alpha W_{11}^{(1)}\left(-\tau_{2 k}\right)+e^{i \omega_{0} \tau_{2 k}} W_{20}^{(2)}(0)+2 e^{-i \omega_{0} \tau_{2 k}} W_{11}^{(2)}(0)\right) \\
& \left.+f_{20}^{(2)}\left(\bar{\alpha} W_{20}^{(2)}(0)+2 \alpha W_{11}^{(2)}(0)\right)\right] \\
& +\overline{\beta^{*}}\left[f_{20}^{(3)}\left(e^{i \omega_{0} \tau_{2 k}} W_{20}^{(1)}\left(-\tau_{2 k}\right)+2 e^{-i \omega_{0} \tau_{2 k}} W_{11}^{(1)}\left(-\tau_{2 k}\right)\right)\right. \\
& +f_{11}^{(3)}\left(\bar{\beta} W_{20}^{(1)}\left(-\tau_{2 k}\right)+2 \beta W_{11}^{(1)}\left(-\tau_{2 k}\right)+e^{i \omega_{0} \tau_{2 k}} W_{20}^{(3)}(0)+2 e^{-i \omega_{0} \tau_{2 k}} W_{11}^{(3)}(0)\right) \\
& \left.\left.+f_{20}^{(3)}\left(\bar{\beta} W_{20}^{(3)}(0)+2 \beta W_{11}^{(3)}(0)\right)\right]\right\} \frac{z^{2} \bar{z}}{2}+\cdots
\end{aligned}
$$

Comparing the coefficients of two sides of (3.14), we have

$$
\begin{aligned}
& g_{20}= \bar{D}\left[\left(f_{200}^{(1)} e^{i \omega_{0} \tau_{1 k}}+2 \alpha f_{110}^{(1)}+2 \beta f_{101}^{(1)}+2 \alpha \beta f_{011}^{(1)} e^{-i \omega_{0} \tau_{1 k}}\right) e^{-i \omega_{0} \tau_{1 k}}\right. \\
&+\overline{\alpha^{*}}\left(f_{20}^{(2)} e^{-2 i \omega_{0} \tau_{2 k}}+f_{02}^{(2)} \alpha^{2}+2 \alpha f_{11}^{(2)} e^{-i \omega_{0} \tau_{2 k}}\right) \\
&\left.+\overline{\beta^{*}}\left(f_{20}^{(3)} e^{-2 i \omega_{0} \tau_{2 k}}+f_{02}^{(3)} \beta^{2}+2 \beta f_{11}^{(3)} e^{-i \omega_{0} \tau_{2 k}}\right)\right], \\
& g_{11}= \bar{D}\left[f_{200}^{(1)}+2 f_{110}^{(1)} \operatorname{Re}\left\{\alpha e^{-i \omega_{0} \tau_{1 k}}\right\}+2 f_{101}^{(1)} \operatorname{Re}\left\{\beta e^{-i \omega_{0} \tau_{1 k}}\right\}+2 f_{011}^{(1)} \operatorname{Re}\{\alpha \bar{\beta}\}\right. \\
&+ \overline{\alpha^{*}}\left(f_{20}^{(2)}+f_{11}^{(2)}\left(\bar{\alpha} e^{-i \omega_{0} \tau_{2 k}}+\alpha e^{i \omega_{0} \tau_{2 k}}\right)+f_{20}^{(2)} \alpha \bar{\alpha}\right) \\
&\left.+\overline{\beta^{*}}\left(f_{20}^{(3)}+f_{11}^{(3)}\left(\bar{\beta} e^{-i \omega_{0} \tau_{2 k}}+\beta e^{i \omega_{0} \tau_{2 k}}\right)+f_{20}^{(3)} \beta \bar{\beta}\right)\right], \\
& g_{02}= \bar{D}\left[\left(f_{200}^{(1)} e^{-i \omega_{0} \tau_{1 k}}+2 \bar{\alpha} f_{110}^{(1)}+2 \bar{\beta} f_{101}^{(1)}+2 \bar{\alpha} \bar{\beta} f_{011}^{(1)} e^{i \omega_{0} \tau_{1 k}}\right) e^{i \omega_{0} \tau_{1 k}}\right. \\
&+ \overline{\alpha^{*}}\left(f_{20}^{(2)} e^{2 i \omega_{0} \tau_{2 k}}+f_{02}^{(2)} \bar{\alpha}^{2}+2 \bar{\alpha} f_{11}^{(2)} e^{i \omega_{0} \tau_{2 k}}\right) \\
&+\left.\overline{\beta^{*}}\left(f_{20}^{(3)} e^{2 i \omega_{0} \tau_{2 k}}+f_{02}^{(3)} \bar{\beta}^{2}+2 \bar{\beta} f_{11}^{(3)} e^{i \omega_{0} \tau_{2 k}}\right)\right], \\
& g_{21}= \bar{D}\left\{f_{200}^{(1)}\left[W_{20}^{(1)}(0)+2 W_{11}^{(1)}(0)\right]\right. \\
&+ f_{110}^{(1)}\left[W_{20}^{(2)}\left(-\tau_{1 k}\right)+2 W_{11}^{(2)}\left(-\tau_{1 k}\right)\right. \\
&\left.\quad+\bar{\alpha} e^{i \omega_{0} \tau_{1 k}} W_{20}^{(1)}(0)+2 \alpha e^{-i \omega_{0} \tau_{1 k}} W_{11}^{(1)}(0)\right] \\
&+ f_{101}^{(1)}\left[W_{20}^{(3)}\left(-\tau_{1 k}\right)+2 W_{11}^{(3)}\left(-\tau_{1 k}\right)\right. \\
&\left.+\bar{\beta} e^{i \omega_{0} \tau_{1 k}} W_{20}^{(1)}(0)+2 \beta e^{-i \omega_{0} \tau_{1 k}} W_{11}^{(1)}(0)\right] \\
&+ f_{011}^{(1)}\left[\bar{\beta} e^{i \omega_{0} \tau_{1 k}} W_{20}^{(2)}\left(-\tau_{1 k}\right)+2 \beta e^{-i \omega_{0} \tau_{1 k}} W_{11}^{(2)}\left(-\tau_{1 k}\right)\right. \\
&+\left.+\bar{\alpha} e^{i \omega_{0} \tau_{1 k}} W_{20}^{(3)}\left(-\tau_{1 k}\right)+2 \alpha e^{-i \omega_{0} \tau_{1 k}} W_{11}^{(3)}\left(-\tau_{1 k}\right)\right] \\
&+ f_{11}^{(2)}\left(\overline { \alpha } W _ { 2 0 } ^ { ( 2 ) } \left(e^{i \omega_{0} \tau_{2 k}} W_{20}^{(1)}\left(-\tau_{2 k}\right)+2 \alpha W_{11}^{(1)}\left(-\tau_{2 k}\right)\right.\right. \\
&\left.-i \omega_{0} \tau_{2 k} W_{11}^{(1)}\left(-\tau_{2 k}\right)\right) \\
&
\end{aligned}
$$




$$
\begin{aligned}
& \left.\quad+e^{i \omega_{0} \tau_{2 k}} W_{20}^{(2)}(0)+2 e^{-i \omega_{0} \tau_{2 k}} W_{11}^{(2)}(0)\right) \\
& \left.+f_{20}^{(2)}\left(\bar{\alpha} W_{20}^{(2)}(0)+2 \alpha W_{11}^{(2)}(0)\right)\right] \\
& +\overline{\beta^{*}}\left[f_{20}^{(3)}\left(e^{i \omega_{0} \tau_{2 k}} W_{20}^{(1)}\left(-\tau_{2 k}\right)+2 e^{-i \omega_{0} \tau_{2 k}} W_{11}^{(1)}\left(-\tau_{2 k}\right)\right)\right. \\
& +f_{11}^{(3)}\left(\bar{\beta} W_{20}^{(1)}\left(-\tau_{2 k}\right)+2 \beta W_{11}^{(1)}\left(-\tau_{2 k}\right)\right. \\
& \left.+e^{i \omega_{0} \tau_{2 k}} W_{20}^{(3)}(0)+2 e^{-i \omega_{0} \tau_{2 k}} W_{11}^{(3)}(0)\right) \\
& \left.\left.+f_{20}^{(3)}\left(\bar{\beta} W_{20}^{(3)}(0)+2 \beta W_{11}^{(3)}(0)\right)\right]\right\} .
\end{aligned}
$$

Since $W_{20}(\theta)$ and $W_{11}(\theta)$ for $\theta \in[-\tau, 0]$ appear in $g_{21}$, we still need to compute them. From (3.6), (3.9) and (3.11), we have

$$
\begin{aligned}
W^{\prime}= & u_{t}^{\prime}-2 \operatorname{Re}\left\{z^{\prime}(t) q(\theta)\right\} \\
& =\left\{\begin{array}{l}
A\left(\tau_{k}\right) W(t, \theta)-2 \operatorname{Re}\left\{\bar{q}^{*}(0) f_{0}(z(t), \bar{z}(t)) q(\theta)\right\}, \\
A\left(\tau_{k}\right) W(t, \theta)-2 \operatorname{Re}\left\{\bar{q}^{*}(0) f_{0}(z(t), \bar{z}(t)) q(0)\right\} \\
+f_{0}(z(t), \bar{z}(t)),
\end{array}\right. \\
& \stackrel{\theta}{\text { def }} A\left(\tau_{k}\right) W(t, \theta)+H(z(t), \bar{z}(t), \theta),
\end{aligned}
$$

where

$$
H(z, \bar{z}, \theta)=H_{20}(\theta) \frac{z^{2}}{2}+H_{11}(\theta) z \bar{z}+H_{02}(\theta) \frac{\bar{z}^{2}}{2}+\cdots .
$$

From (3.15) and (3.16), we have

$A\left(\tau_{k}\right) W(t, \theta)-W^{\prime}=-H(z, \bar{z}, \theta)=-H_{20}(\theta) \frac{z^{2}}{2}-H_{11}(\theta) z \bar{z}-H_{02}(\theta) \frac{\bar{z}^{2}}{2}-\cdots$.

In view of $(3.10)$, one can obtain

$$
A\left(\tau_{k}\right) W(t, \theta)=A\left(\tau_{k}\right) W_{20}(\theta) \frac{z^{2}}{2}+A\left(\tau_{k}\right) W_{11}(\theta) z \bar{z}+\cdots
$$

and

$$
W^{\prime}=W_{z} z^{\prime}+W_{\bar{z}} \bar{z}^{\prime}=2 i \omega_{0} W_{20}(\theta) \frac{z^{2}}{2}+\cdots
$$

It follows from (3.18) and (3.19) that

$$
A\left(\tau_{k}\right) W(t, \theta)-W^{\prime}=\left[A\left(\tau_{k}\right)-2 i \omega_{0} I\right] W_{20}(\theta) \frac{z^{2}}{2}+A\left(\tau_{k}\right) W_{11}(\theta) z \bar{z}+\cdots .
$$

Thus, (3.17) and (3.20) imply that

$$
\left[A\left(\tau_{k}\right)-2 i \omega_{0} I\right] W_{20}(\theta) \frac{z^{2}}{2}+A\left(\tau_{k}\right) W_{11}(\theta) z \bar{z}+\cdots
$$




$$
=-H_{20}(\theta) \frac{z^{2}}{2}-H_{11}(\theta) z \bar{z}-H_{02}(\theta) \frac{\bar{z}^{2}}{2}-\cdots .
$$

Comparing the coefficients of two sides of (3.21), we have

(3.22) $\left[A\left(\tau_{k}\right)-2 i \omega_{0} I\right] W_{20}(\theta)=-H_{20}(\theta)$ and $A\left(\tau_{k}\right) W_{11}(\theta)=-H_{11}(\theta)$.

From (3.15), we know that for $\theta \in[-\tau, 0)$,

$$
\begin{aligned}
H(z, \bar{z}, \theta) & =-2 \operatorname{Re}\left\{\bar{q}^{*}(0) f_{0}(z(t), \bar{z}(t)) q(\theta)\right\} \\
& =-\bar{q}^{*}(0) f_{0}(z(t), \bar{z}(t)) q(\theta)-q^{*}(0) \overline{f_{0}}(z(t), \bar{z}(t)) \bar{q}(\theta) \\
& =-g(z, \bar{z}) q(\theta)-\bar{g}(z, \bar{z}) \bar{q}(\theta) \\
& =-\left(g_{20} q(\theta)+\bar{g}_{02} \bar{q}(\theta)\right) \frac{z^{2}}{2}-\left(g_{11} q(\theta)+\bar{g}_{11} \bar{q}(\theta)\right) z \bar{z}+\cdots
\end{aligned}
$$

Comparing the coefficients with (3.16) gives that

$$
H_{20}(\theta)=-\left(g_{20} q(\theta)+\bar{g}_{02} \bar{q}(\theta)\right)
$$

and

$$
H_{11}(\theta)=-\left(g_{11} q(\theta)+\bar{g}_{11} \bar{q}(\theta)\right)
$$

From (3.22), (3.24) and the definition of $A\left(\tau_{k}\right)$, we have

$$
W_{20}^{\prime}(\theta)=2 i \omega_{0} W_{20}(\theta)+g_{20} q(\theta)+\bar{g}_{02} \bar{q}(\theta) .
$$

Note that $q(\theta)=q(0) e^{i \omega_{0} \theta}$, hence

$$
W_{20}(\theta)=\frac{i g_{20}}{\omega_{0}} q(\theta)+\frac{i \bar{g}_{02}}{3 \omega_{0}} \bar{q}(\theta)+E_{1} e^{2 i \omega_{0} \theta} .
$$

Similarly, from (3.22), (3.25) and the definition of $A\left(\tau_{k}\right)$, we have

$$
W_{11}^{\prime}(\theta)=g_{11} q(\theta)+\bar{g}_{11} \bar{q}(\theta)
$$

and

$$
W_{11}(\theta)=-\frac{i g_{11}}{\omega_{0}} q(\theta)+\frac{i \bar{g}_{11}}{\omega_{0}} \bar{q}(\theta)+E_{2} .
$$

In what follows, we shall seek appropriate $E_{1}$ and $E_{2}$ in (3.26) and (3.27), respectively. It follows from the definition of $A\left(\tau_{k}\right)$ and (3.22) that

$$
\int_{-\tau_{k}}^{0} d \eta(\theta) W_{20}(\theta)=2 i \omega_{0} W_{20}(0)-H_{20}(0)
$$

and

$$
\int_{-\tau_{k}}^{0} d \eta(\theta) W_{11}(\theta)=-H_{11}(0)
$$

Note that $q(\theta)$ is the eigenvector of $A\left(\tau_{k}\right)$ and from (3.26) and the definition of $A\left(\tau_{k}\right)$, we know that

$$
\int_{-\tau_{k}}^{0} d \eta(\theta) W_{20}(\theta)=-g_{20} q(0)+\frac{\bar{g}_{02}}{3} \bar{q}(0)+\int_{-\tau_{k}}^{0} d \eta(\theta) e^{2 i \omega_{0} \theta} E_{1}
$$


and

$$
2 i \omega_{0} W_{20}(0)=-2 g_{20} q(0)-\frac{2 \bar{g}_{02}}{3} \bar{q}(0)+2 i \omega_{0} E_{1} .
$$

Thus, (3.28) becomes

$$
-g_{20} q(0)-\bar{g}_{02} \bar{q}(0)+\left(2 i \omega_{0} I-\int_{-\tau_{k}}^{0} d \eta(\theta) e^{2 i \omega \widetilde{\tau} \theta}\right) E_{1}=H_{20}(0) .
$$

Similarly, from (3.27), we have

$$
\int_{-\tau_{k}}^{0} d \eta(\theta) W_{11}(\theta)=g_{11} q(0)+\bar{g}_{11} \bar{q}(0)+\int_{-\tau_{k}}^{0} d \eta(\theta) E_{2}
$$

Hence (3.29) becomes

$$
-\left(g_{11} q(0)+\bar{g}_{11} \bar{q}(0)\right)-\int_{-\tau_{k}}^{0} d \eta(\theta) E_{2}=H_{11}(0) .
$$

From (3.15) and (3.16), we know that

$$
\begin{aligned}
H(z, \bar{z}, 0)= & H_{20}(0) \frac{z^{2}}{2}+H_{11}(0) z \bar{z}+H_{02}(0) \frac{\bar{z}^{2}}{2}+\cdots \\
= & -2 \operatorname{Re}\left\{\bar{q}^{*}(0) f_{0}(z(t), \bar{z}(t)) q(0)\right\}+f_{0}(z(t), \bar{z}(t)) \\
= & -\left(g_{20} q(0)+\bar{g}_{02} \bar{q}(0)\right) \frac{z^{2}}{2}-\left(g_{11} q(0)+\bar{g}_{11} \bar{q}(0)\right) z \bar{z} \\
& +f_{0}(z(t), \bar{z}(t))+\cdots
\end{aligned}
$$

It follows together with (3.21) that

$$
H_{20}(0)=-\left(g_{20} q(0)+\bar{g}_{02} \bar{q}(0)\right)+h_{20}
$$

and

$$
H_{11}(0)=-\left(g_{11} q(0)+\bar{g}_{11} \bar{q}(0)\right)+h_{11},
$$

where

$$
\begin{array}{r}
h_{20}=\left(\begin{array}{c}
\left(f_{200}^{(1)} e^{i \omega_{0} \tau_{1 k}}+2 \alpha f_{110}^{(1)}+2 \beta f_{101}^{(1)}+2 \alpha \beta f_{011}^{(1)} e^{-i \omega_{0} \tau_{1 k}}\right) e^{-i \omega_{0} \tau_{1 k}} \\
f_{20}^{(2)} e^{-2 i \omega_{0} \tau_{2 k}}+f_{02}^{(2)} \alpha^{2}+2 \alpha f_{11}^{(2)} e^{-i \omega_{0} \tau_{2 k}} \\
f_{20}^{(3)} e^{-2 i \omega_{0} \tau_{2 k}}+f_{02}^{(3)} \beta^{2}+2 f_{11}^{(3)} \beta e^{-i \omega_{0} \tau_{2 k}}
\end{array}\right), \\
h_{11}=\left(\begin{array}{c}
f_{200}^{(1)}+2 f_{110}^{(1)}\left\{\alpha e^{\left.-i \omega_{0} \tau_{1 k}\right\}}+2 f_{101}^{(1)} \operatorname{Re}\left\{\beta e^{-i \omega_{0} \tau_{1 k}}\right\}+2 f_{011}^{(1)} \operatorname{Re}\{\alpha \bar{\beta}\}\right. \\
f_{11}^{(2)}\left(\bar{\alpha} e^{-i \omega_{0} \tau_{2 k}}+\alpha e^{i \omega_{0} \tau_{2 k}}\right)+f_{20}^{(2)}+f_{02}^{(2)} \alpha \bar{\alpha} \\
f_{11}^{(3)}\left(\bar{\beta} e^{-i \omega_{0} \tau_{2 k}}+\beta e^{i \omega_{0} \tau_{2 k}}\right)+f_{20}^{(3)}+f_{02}^{(3)} \beta \bar{\beta}
\end{array}\right) .
\end{array}
$$

Substituting (3.36) into (3.32), we obtain

$$
\left(2 i \omega_{0} I-\int_{-\tau_{k}}^{0} d \eta(\theta) e^{2 i \omega_{0} \theta}\right) E_{1}=h_{20}
$$


that is

$$
\left(\begin{array}{ccc}
2 i \omega_{0}-a_{11} & -a_{12} e^{-2 i \omega_{0} \tau_{1 k}} & -a_{13} e^{-2 i \omega_{0} \tau_{1 k}} \\
-a_{21} e^{-2 i \omega_{0} \tau_{2 k}} & 2 i \omega_{0} & 0 \\
-a_{31} e^{-2 i \omega_{0} \tau_{2 k}} & 0 & 2 i \omega_{0}-a_{33}
\end{array}\right) E_{1}=h_{20} .
$$

Solving this equation, one can obtain

$$
E_{1}=\left(\begin{array}{ccc}
2 i \omega_{0}-a_{11} & -a_{12} e^{-2 i \omega_{0} \tau_{1 k}} & -a_{13} e^{-2 i \omega_{0} \tau_{1 k}} \\
-a_{21} e^{-2 i \omega_{0} \tau_{2 k}} & 2 i \omega_{0} & 0 \\
-a_{31} e^{-2 i \omega_{0} \tau_{2 k}} & 0 & 2 i \omega_{0}-a_{33}
\end{array}\right)^{-1} h_{20}
$$

Similarly, substituting (3.37) into (3.33), we get

$$
\int_{-\tau_{k}}^{0} d \eta(\theta) E_{2}=-h_{11}
$$

which means that

$$
\left(\begin{array}{ccc}
-a_{11} & -a_{12} & -a_{13} \\
-a_{21} & 0 & 0 \\
-a_{31} & 0 & -a_{33}
\end{array}\right) E_{2}=h_{11}
$$

From this equation, we can get

$$
E_{2}=\left(\begin{array}{ccc}
-a_{11} & -a_{12} & -a_{13} \\
-a_{21} & 0 & 0 \\
-a_{31} & 0 & -a_{33}
\end{array}\right)^{-1} h_{11}
$$

Thus $g_{21}$ can be expressed explicitly and we now can easily evaluate the following values

$$
\begin{aligned}
c_{1}\left(\tau_{k}\right) & =\frac{i}{2 \omega_{0}}\left(g_{11} g_{20}-2\left|g_{11}\right|^{2}-\frac{1}{3}\left|g_{02}\right|^{2}\right)+\frac{g_{21}}{2} \\
\mu_{2} & =-\frac{\operatorname{Re}\left\{c_{1}\left(\tau_{k}\right)\right\}}{\operatorname{Re}\left\{\lambda^{\prime}\left(\tau_{k}\right)\right\}} \\
\beta_{2} & =2 \operatorname{Re}\left\{c_{1}\left(\tau_{k}\right)\right\} \\
T_{2} & =-\frac{\operatorname{Im}\left\{c_{1}\left(\tau_{k}\right)\right\}+\mu_{2} \operatorname{Im}\left\{\lambda^{\prime}\left(\tau_{k}\right)\right\}}{\omega_{0}}
\end{aligned}
$$

which determine the properties of bifurcating periodic solutions at the critical value $\tau_{k}$. More precisely, $\mu_{2}$ determines the direction of Hopf bifurcation, $\beta_{2}$ determines the stability of bifurcating periodic solution and $T_{2}$ determines the period of the bifurcating periodic solution. Therefore, summarizing the above discussions, we have the following main result. 


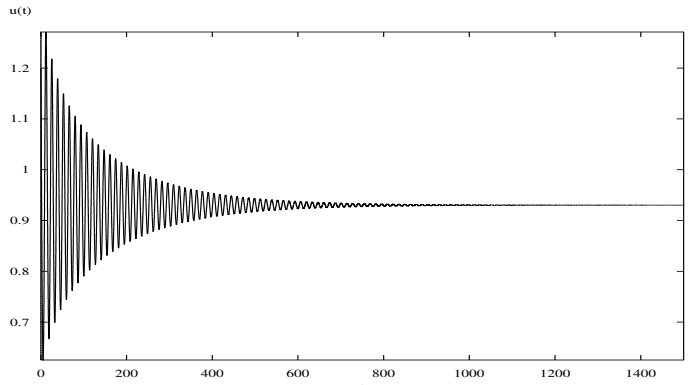

Figure 1. The trajectory graph in $t$ - $u$ plane of the system (4.1) with $\tau_{1}=0.6, \tau_{2}=0.58$, and initial data $u(t)=0.93$, $v(t)=2.09, w(t)=0.3$ for $t \in[-1.18,0]$.

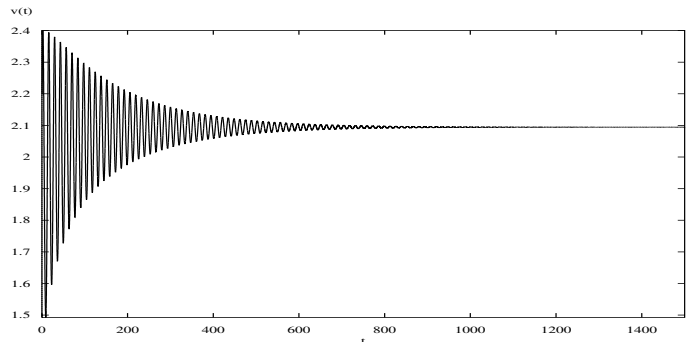

Figure 2. The trajectory graph in $t-v$ plane of the system (4.1) with $\tau_{1}=0.6, \tau_{2}=0.58$, and initial data $u(t)=$ $0.93, v(t)=2.09, w(t)=0.3$ for $t \in[-1.18,0]$.

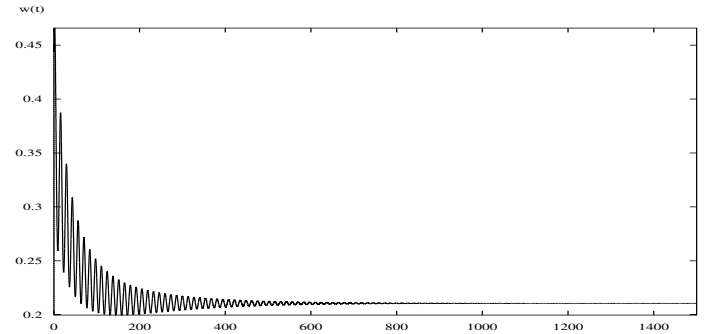

Figure 3. The trajectory graph in $t-w$ plane of the system (4.1) with $\tau_{1}=0.6, \tau_{2}=0.58$, and initial data $u(t)=$ $0.93, v(t)=2.09, w(t)=0.3$ for $t \in[-1.18,0]$. 
Theorem 3.1. If $\mu_{2}>0\left(\mu_{2}<0\right)$, then the Hopf bifurcation is supercritical (subcritical) and the bifurcating periodic solutions exist for $\tau>\tau_{k}\left(\tau<\tau_{k}\right)$. If $\beta_{2}<0\left(\beta_{2}>0\right)$, then the periodic solutions are stable (unstable). If $T_{2}>$ $0\left(T_{2}<0\right)$, then the period of the bifurcating periodic solutions of system $(1.2)$ increases (decreases).

Remark 3.2. If Hopf bifurcations in Theorem 3.1 are supercritical (subcritical), that is, the bifurcating periodic solutions exist for $\tau>\tau_{k}\left(\tau<\tau_{k}\right)$, then Theorem 3.1 implies that the period of the bifurcating periodic solutions increases (decreases) with $\tau$ keeping monotonically increasing (decreasing) away from $\tau_{k}$ if $T_{2}>0\left(T_{2}<0\right)$.

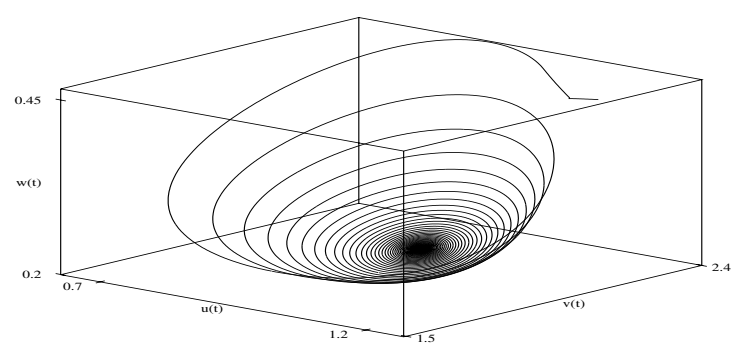

FiguRE 4. The phase graphs of the system (4.1) with $\tau_{1}=0.6$ and $\tau_{2}=0.58$.

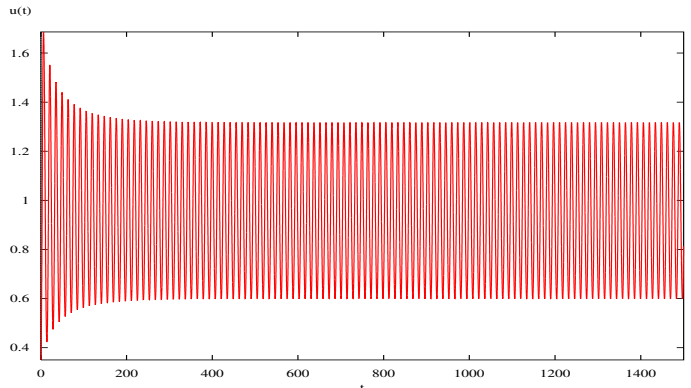

FIGURE 5. The trajectory graph in $t$ - $u$ plane of the system (4.1) with $\tau_{1}=0.76, \tau_{2}=0.66$, and initial data $u(t)=$ $0.35, v(t)=1.5, w(t)=0.18$ for $t \in[-1.42,0]$. 


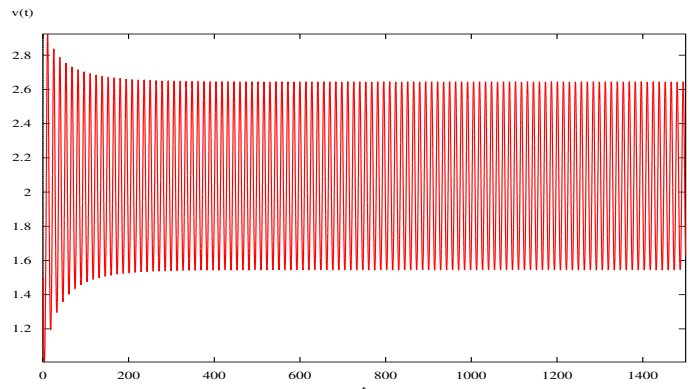

Figure 6 . The trajectory graph in $t-v$ plane of the system (4.1) with $\tau_{1}=0.76, \tau_{2}=0.66$, and initial data $u(t)=$ $0.35, v(t)=1.5, w(t)=0.18$ for $t \in[-1.42,0]$.

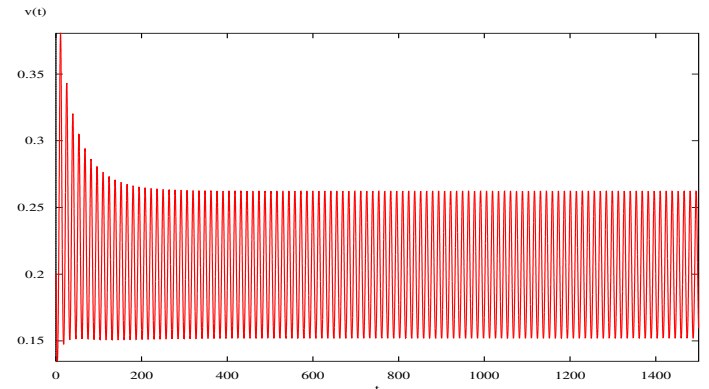

Figure 7 . The trajectory graph in $t-w$ plane of the system (4.1) with $\tau_{1}=0.76, \tau_{2}=0.66$, and initial data $u(t)=$ $0.35, v(t)=1.5, w(t)=0.18$ for $t \in[-1.42,0]$.

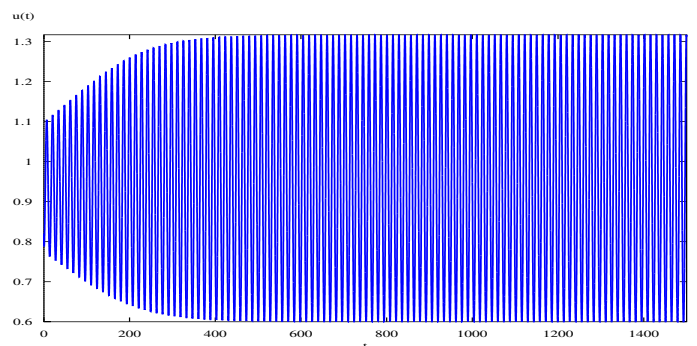

FiguRE 8 . The trajectory graph in $t$ - $u$ plane of the system (4.1) with $\tau_{1}=0.76, \tau_{2}=0.66$, and initial data $u(t)=$ $0.82, v(t)=2.2, w(t)=0.22$ for $t \in[-1.42,0]$. 


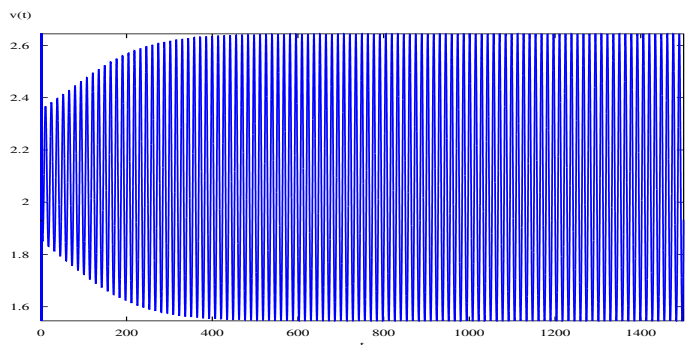

FIGURE 9. The trajectory graph in $t-v$ plane of the system (4.1) with $\tau_{1}=0.76, \tau_{2}=0.66$, and initial data $u(t)=$ $0.82, v(t)=2.2, w(t)=0.22$ for $t \in[-1.42,0]$.

\section{Discussions and numerical simulations}

In the present paper, by regarding the sum $\tau$ of two delays $\tau_{1}$ and $\tau_{2}$ as the bifurcation parameter, we have studied the effect of the time delay $\tau$ on the dynamical behaviors near the positive equilibrium of the system (1.2). From Section 2, we know that under the hypotheses (H1) and (H2), the positive equilibrium $E^{*}\left(u^{*}, v^{*}, w^{*}\right)$ of the system (1.2) is asymptotically stable when $\tau \in\left[0, \tau_{0}\right)$. Furthermore, if $g^{\prime}\left(\omega_{0}^{2}\right) \neq 0$, then $E^{*}$ loses its stability and a supercritical Hopf bifurcation occurs when $\tau$ increases and crosses through the critical value $\tau_{0}$. In particular, by applying the normal form theory and the center manifold reduction for FDEs, an explicit algorithm determining the direction of Hopf bifurcations and the stability of bifurcating periodic solutions has been also obtained.

In this section, we give some numerical simulations for a special case of the system (1.2) to support our analytical results obtained in Sections 2 and 3. As an example, we consider system (1.2) with the coefficients $r=1.3, K=2.5$, $a=0.45, b=0.3, A=0.5, B=0.35, d=D=0.4, e=0.55, G=0.1$ and $E=0.6$, that is

$$
\text { (4.1) }\left\{\begin{array}{l}
u^{\prime}(t)=1.3 u(t)\left(1-\frac{u(t)}{2.5}\right)-\frac{0.45 u(t) v\left(t-\tau_{1}\right)}{1+0.3 u(t)}-\frac{0.5 u(t) w\left(t-\tau_{1}\right)}{1+0.35 u(t)}, \\
v^{\prime}(t)=v(t)\left(-0.4+\frac{0.55 u\left(t-\tau_{2}\right)}{1+0.3 u\left(t-\tau_{2}\right)}\right) \\
w^{\prime}(t)=w(t)\left(-0.4-0.1 w(t)+\frac{0.6 u\left(t-\tau_{2}\right)}{1+0.35 u\left(t-\tau_{2}\right)}\right) .
\end{array}\right.
$$

It is easy to verify that the conditions (H1) and (H2) hold and therefore the system (4.1) has a unique positive equilibrium $E^{*}\left(u^{*}, v^{*}, w^{*}\right)=(0.9302,2.0945$, $0.2105)$. In addition, one can obtain that $\omega_{0}=0.4623$ and $\tau_{0}=1.2657$. Thus, we know from Theorem 2.4 that $E^{*}$ is asymptotically stable when $0 \leq \tau<$ 
1.2657 and unstable when $\tau>1.2657$. The numerical simulations for system (4.1) with $\tau_{1}=0.6$ and $\tau_{1}=0.58$ are shown by Figs.1-4, and $\tau_{1}=0.76$ and $\tau_{2}=0.66$ are shown by Figs. $5-11$.

On the other hand, $g(z)$ defined in Section 2 has the form

$$
g(z)=z^{3}+0.0925 z^{2}-0.0663 z-0.000023536 .
$$

Therefore, $g^{\prime}\left(\omega_{0}^{2}\right)=0.1103 \neq 0$. It follows from Theorem 2.3 that when $\tau_{1}$ and $\tau_{2}$ are increased and pass through the critical value $\tau_{0}=1.2657, E^{*}$ loses its stability and a Hopf bifurcation occurs. In addition, when $\tau=\tau_{1}+\tau_{2}=$ $\tau_{0}=1.2657$, we can obtain $c_{1}(0)=-0.1114+0.1845 i$. Hence, the Hopf bifurcation occurring at the first bifurcation point $\tau_{0}=1.2657$ is supercritical and the bifurcating periodic solution is orbitally asymptotically stable on the center manifold. The numerical simulations to system (4.1) with $\tau_{1}=0.76$ and $\tau_{1}=0.66$ are shown by Figs.5-11.

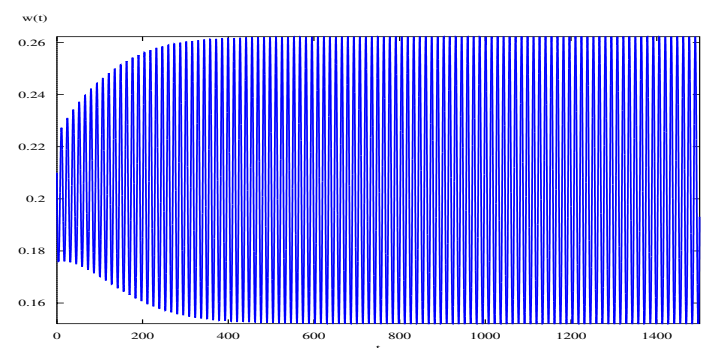

Figure 10. The trajectory graph in $t$ - $w$ plane of the system (4.1) with $\tau_{1}=0.76, \tau_{2}=0.66$, and initial data $u(t)=$ $0.82, v(t)=2.2, w(t)=0.22$ for $t \in[-1.42,0]$.

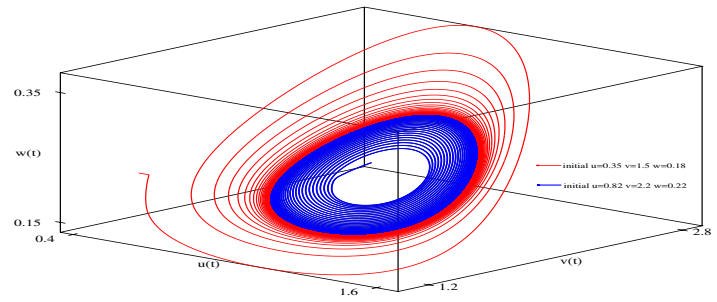

FIGURE 11. The phase graphs of the system (4.1) with $\tau_{1}=$ 0.76 and $\tau_{2}=0.66$. 
Acknowledgement. The authors are grateful to the anonymous referees for their helpful comments and valuable suggestions.

\section{References}

[1] H. Boudjellaba and T. Sari, Stability loss delay in harvesting competing populations, J. Differential Equations 152 (1999), no. 2, 394-408.

[2] G. J. Butler and P. Waltman, Bifurcation from a limit cycle in a two predator-one prey ecosystem modeled on a chemostat, J. Math. Biol. 12 (1981), no. 3, 295-310.

[3] J. M. Cushing, Periodic two-predator, one-prey interactions and the time sharing of a resource niche, SIAM J. Appl. Math. 44 (1984), no. 2, 392-410.

[4] O. Diekmann, R. Nisbet, W. Gurney, and F. Bosch, Simple mathematical models for cannibalism: a critique and a new approach, Math. Biosci. 78 (1986), no. 1, 21-46.

[5] H. I. Freedman, Deterministic Mathematical Models in Population Ecology, Marcel Dekker, New York, 1980.

[6] J. K. Hale, Theory of Functional Differential Equations, Spring-Verlag, New York, 1977.

[7] B. D. Hassard, N. D. Kazarinoff, and Y. H. Wan, Theory and Applications of Hopf bifurcation, Cambridge University Press, Cambridge, 1981.

[8] S.-B. Hsu, S. P. Hubbell, and P. Waltman, Competing predators, SIAM J. Appl. Math. 35 (1978), no. 4, 617-625.

[9] T. K. Kar and A. Batabyalb, Persistence and stability of a two prey one predator system, International Journal of Engineering, Science and Technology 2 (2010), 174-190.

[10] A. J. Lotka, Elements of Physical Biology, New York, Williams and Wilkins, 1925

[11] Z. Liu and R. Yuan, Stability and bifurcation in a harmonic oscillator with delays, Chaos Solitons Fractals 23 (2005), no. 2, 551-562.

[12] Z. Lu and X. Liu, Analysis of a predator-prey model with modified Holling-Tanner functional response and time delay, Nonlinear Anal. Real World Appl. 9 (2008), no. 2, 641-650.

[13] S. Muratori and S. Rinaldi, Remarks on competitive coexistence, SIAM J. Appl. Math. 49 (1989), no. 5, 1462-1472.

[14] J. D. Murray, Mathematical Biology, Biomathematics 19, Sringer-Verlag, Berlin Heidelberg, 1989.

[15] B. Patra, A. Maiti, and G. P. Samanta, Effect of time-delay on a ratio-dependent food chain model, Nonlinear Anal. Model. Control 14 (2009), no. 2, 199-216.

[16] S. Ruan, A. Ardito, P. Ricciardi, and D. DeAngelis, Coexistence in competition models with density-dependent mortality, Comptes Rend. Biol 330 (2007), 845-854.

[17] H. L. Smith, The interaction of steady state and Hopf bifurcations in a two-predatorone-prey competition model, SIAM J. Appl. Math. 42 (1982), no. 1, 27-43.

[18] Y. Song and S. Yuan, Bifurcation analysis in a predator-prey system with time delay, Nonlinear Anal. Real World Appl. 7 (2006), no. 2, 265-284.

[19] Y. Takeuchi, Global Dynamical Propertices of Lotka-Volterra Systems, World Scientific, Singapore, 1996.

[20] V. Volterra, Variazionie fluttuazioni del numero dindividui in specie animali conviventi, Mem. Acad. Licei 2 (1926), 31-113.

[21] J. Wei and S. Ruan, Stability and bifurcation in a neural network model with two delays, Phys. D 130 (1999), no. 3-4, 255-272.

[22] J. Wei and M. Li, Global existence of periodic solutions in a tri-neuron network model with delays, Phys. D 198 (2004), no. 1-2, 106-119.

[23] D. Xiao and W. Li, Stability and bifurcation in a delayed ratio-dependent predator-prey system, Proc. Edinb. Math. Soc. (2) 46 (2003), no. 1, 205-220. 
[24] Y. Xu, Q. Gan, and Z. Ma, Stability and bifurcation analysis on a ratio-dependent predator-prey model with time delay, J. Comput. Appl. Math. 230 (2009), no. 1, 187203.

[25] X. Yan, Stability and Hopf bifurcation for a delayed prey-predator system with diffusion effects, Appl. Math. Comput. 192 (2007), no. 2, 552-566.

\section{GuO-Hu Cui}

Department of Mathematics

LANZHOU JIAOTONG UNIVERSITY

Lanzhou 730070, P. R. China

AND

Teaching and Research Group of Mathematics

The First Middle school of TongWei

TongWei, 743300, P. R. China

E-mail address: cuigh1689@126.com

Xiang-Ping Yan

Department of Mathematics

LANZHOU JIAOTONG UNIVERSITY

Lanzhou 730070, P. R. China

E-mail address: xpyan72@163.com 